\title{
The effects of aging on semen parameters and sperm DNA fragmentation
}

\author{
Víctor Pino ${ }^{1}$, Antonia Sanz ${ }^{1}$, Nicolás Valdés², Javier Crosby³, Antonio Mackenna ${ }^{4}$ \\ ${ }^{1}$ Faculty of Medicine, University of Los Andes, Santiago, Chile. \\ ${ }^{2}$ Environmental Chemist, Department of Epidemiology, Faculty of Obstetrics and Medicine, University of Los \\ Andes, Santiago, Chile. \\ ${ }^{3}$ Biologist and Doctorate in Science, Unit of Reproductive Medicine, Department of Obstetrics and Gynecology, \\ Clínica Las Condes, Santiago, Chile. \\ ${ }^{4}$ Unit of Reproductive Medicine, Department of Obstetrics and Gynecology, Clínica Las Condes, Santiago, Chile.
}

\begin{abstract}
Introduction: This study aimed to look into possible correlations between male age and different sperm parameters derived from semen analysis and sperm deoxyribonucleic acid (DNA) fragmentation.

Methods: This retrospective descriptive study included 2681 male patients who underwent semen analysis at Clínica Las Condes (CLC), Santiago, Chile, between January 2014 and May 2017; correlations between age and sperm parameters were analyzed.

Results: Males above the age of 50 were significantly more likely to present anomalies in semen volume, sperm concentration, and sperm DNA fragmentation; males aged $41+$ years were more likely to have lower sperm concentration levels; males aged $31+$ years were more likely to have decreased sperm motility; when concentration was constant, more volume and motility anomalies were seen as age increased; when volume was kept constant, more motility and concentration anomalies were seen as age increased; and when motility was constant, normal semen volumes decreased as age increased.
\end{abstract}

Conclusion: Our study showed that male age significantly affects sperm parameters that might have an impact on male fertility.

Keywords: fertility, infertility, paternal age, aging, semen analysis, sperm DNA fragmentation

\section{INTRODUCTION}

Aging is a natural inevitable process that affects every individual and introduces a series of physiological changes in our bodies. One of the changes associated with aging is decreased reproductive capacity (Gunes et al., 2016). Studies on this topic often focus on the impacts of aging on female fertility, a matter assigned greater clinical relevance, and generally neglect how aging affects male fertility. This has led many to believe that aging has negligible effects on male reproductive capacity, and that men would therefore have a nearly endless reproductive lifespan. However, several studies demonstrated a direct correlation between aging and structural and functional changes of sexual organs and the endocrine system, which by their turn suggest an effect on sperm parameters and fertility (Gunes et al., 2016; Johnson et al., 2015).

Aging affects the male sexual organs in different ways. The volume of the testes starts to decrease after 60 years of age. Gonadotropin levels increase and testosterone levels decrease with aging. The number of Leydig, Sertoli, and germ cells decreases with aging. Aging introduces vascular changes that lead to testicular fibrosis. Aging has also been associated with increased incidence of benign prostatic hyperplasia, which affects ejaculation and semen volume (Mahmoud et al., 2003; Zitzmann, 2013; Paniagua et al., 1986; Neaves et al., 1984).

Studies show that semen parameters are negatively affected by aging (Oliveira et al., 2014). Decreases in semen volume caused by impaired accessory gland function, and decreased daily sperm production, total sperm count, and sperm viability have also been linked to aging (Gunes et al., 2016). Some authors also suggested that sperm morphology alterations occur from the age of 40 years (Neaves et al., 1984; Johnson et al., 1984).

Aging has been correlated with increased oxidative stress, which leads to increased lipid peroxidation and formation of reactive oxygen species (ROS) in the mitochondria (Gunes et al., 2016). The body's antioxidant capacity decreases, and oxidative damage to spermatic DNA becomes more likely. In later stages, oxidative stress may lead to cell death and decrease sperm fertilization capacity. It may also cause genetic mutations in the gametes, thereby increasing the probability of miscarriage, genetic mutations in the offspring, and metabolic, psychiatric, and neurological disorders (Kong et al., 2012). Male germ cells undergo continuous DNA replication and division throughout an individual's life. Therefore, older men are at greater risk of having germ cells carrying mutations (Gunes et al., 2016). Base substitutions account for most of the mutations and cause monogenic disorders such as achondroplasia and Apert Syndrome (Goriely \& Wilkie, 2012). Hypermethylation and hypomethylation of certain DNA segments - events associated with increased incidence of schizophrenia and bipolar disorder - occur more frequently in individuals of advanced age (Goriely \& Wilkie, 2012; Crow, 2000). Furthermore, telomere length generally increases with age (Eisenberg, 2011).

Considering the relevance of this topic vis-à-vis the demographic changes in progress and the insufficient attention given to it by reproductive medicine and society in general, this study aimed to identify specific changes produced by aging on semen parameters and sperm DNA integrity that might affect male fertility. The information discussed herein has use not only in the determination of couple fertility prospects, but also in the education of the general public about aging and fertility.

\section{MATERIALS AND METHODS}

This descriptive retrospective study looked into the data from the charts of patients aged 18 years or older submitted to semen analysis at the Andrology Laboratory of Clínica las Condes in Santiago, Chile, between January 2014 and May 2017. Only the data from the more recent sample were considered for patients with more than one sample recorded in the database. The study included data from 2678 men. All 
samples were collected through masturbation after three to 15 days of abstinence. Sperm analysis and examination were carried out according to the techniques established by the World Health Organization (World Health Organization, 2010). Sperm DNA fragmentation was quantified via the Halosperm ${ }^{\circledR}$ technique (Fernandez et al., 2003). Patients in abstinence for less than three or more than 15 days prior to semen analysis and individuals presenting more than $1,000,000$ round cells (increased round cell count is indicative of seminal tract infection and potentially altered spermatic characteristics for causes other than age, working therefore as a confounding factor) were excluded. Included patients gave consent to joining the study before examination. The consent form had been previously approved by the institution's Ethics Committee.

The variables considered in this study were age and the following semen parameters: volume $(\mathrm{ml})$; sperm concentration (millions/ml); total sperm count (millions); sperm progressive motility $A+B(\%)$; sperm morphology (\% normality); and sperm DNA fragmentation (\%). Normal values were defined based on the WHO standards (World Health Organization, 2010) (Table 1).

The patients were divided into four groups based on age. The age groups were divided as follows: $21-30$ years; $31-40$ years; $41-50$ years; and more than 50 years.

The variables were assessed according to age using logistic regression analysis. Age was first considered a discrete quantitative variable and was later deemed a categorical variable, according to age ranges, in which individuals with ages between 21 and 30 years were set as the reference group. Odds ratios (OR) and their respective $p$ values were calculated based on these analyses. Statistical significance was defined as a $p$ value $\leq 0.05$, and confidence intervals were set at $95 \%$.

Correlations between age and the studied variables were searched and stratified according to the previous results and indicators mentioned above. Statistical analysis was performed on software package Stata release 15. (College Station, TX: StataCorp LLC, 2017).

\section{RESULTS}

Our study included the data from 2678 men with ages averaging $39.2 \pm 6$ years. A total of 119 individuals were aged $21-30$ years; 1579 were aged $31-40$ years; 852 were aged $41-50$ years; and 128 were aged $50+$ years. Table 2 shows the descriptive statistics for semen analysis.

\section{Semen volume}

The risk of presenting anomalous semen volumes increased with age. This finding was statistically significant among men older than 50 years (OR: $2.2 ; 95 \%$ CI [1.11-4.34]; $p=0.022$ ). Men above the age of 50 were 2.2 times more likely to present decreased semen volumes than males aged $21-30$ years.

Table 1. Normal values for semen analysis according to the WHO*

\begin{tabular}{|l|c|}
\hline Variable & Cut-off value \\
\hline Sperm volume & $>1.5 \mathrm{ml}$ \\
\hline Sperm concentration & $>15$ million / ml \\
\hline Total sperm count & $>39$ million \\
\hline Sperm progressive motility $(\mathrm{A}+\mathrm{B})$ & $>32 \%$ \\
\hline Sperm morphology & $>4 \%$ \\
\hline Sperm DNA fragmentation & $<30 \%$ \\
\hline Non-sperm cells & $<1$ million / $\mathrm{ml}$ \\
\hline
\end{tabular}

WHO laboratory manual for the examination and processing of human semen. Geneva: World Health Organization; 2010.

\section{Sperm concentration}

The risk of presenting anomalous sperm concentrations increased with age. This finding was statistically significant among men older than 50 years (OR: 2.09 ; 95\% CI [1.08$4.02] ; p=0.027)$. Men above the age of 50 were 2.09 times more likely to present anomalous sperm concentrations than males aged $21-30$ years.

\section{Total sperm count}

The risk of presenting decreased sperm counts in semen analysis increased with age. This finding became statistically significant from 41 years of age. Men aged 41-50 years were 2.92 times more likely to present decreased sperm counts than males aged $21-30$ years (OR: $2.92 ; 95 \%$ CI [1.16-7.38]; $p=0.023$ ). Men above the age of 50 were 6.15 times more likely to present decreased sperm counts than males aged $21-30$ years (OR: $6.15 ; 95 \%$ CI [2.26-16.73]; $p=0.0001$ ).

\section{Sperm progressive motility $(A+B)$}

Progressive motility decreased as age increased. All age ranges presented statistically significant impairments, unlike the rest of the examined parameters. Group aged 31-40 years - OR: $3.24 ; 95 \%$ CI [1.17-8.94]; $p=0.023 ;$ group aged $41-50$ years - OR: $5.24 ; 95 \%$ CI [1.89-14.52]; $p=0.001 ;$ group aged 50 or older - OR: $11.91 ; 95 \%$ CI [4.04-35.07]; $p<0.0001$; all comparisons versus the group aged $21-30$ years.

\section{Sperm morphology}

No statistically significant correlation was found when patients were analyzed for age versus sperm morphology.

\section{Sperm DNA fragmentation}

The risk of presenting anomalous levels of DNA fragmentation increased with age. This finding was statistically significant among men older than 50 years old (OR: 4.58; $95 \%$ CI [1.16-17.99]; $p=0.029)$. Men above the age of 50 were 4.58 times more likely to present sperm DNA fragmentation than males aged $21-30$ years.

Tables 3, 4 and 5 show the descriptive statistics for sperm volume, concentration, and motility at a constant semen volume.

\section{Motility and sperm concentration at a constant semen volume}

Table 3 shows that, at a constant semen volume, motility decreased as age increased (OR: 1.06; 95\% CI [1.03-1.08]; $p<0.0001)$. Sperm concentration also decreased as age increased (OR: $1.03 ; 95 \%$ CI [1.01-1.05]; $p=0.003$ ). Therefore, each year the risk of presenting motility disorders increased 1.06 time over the previous year. As for sperm concentration at a constant volume, the risk of presenting decreased sperm concentration increased by 1.03 time over the previous year.

\section{Semen volume and sperm motility at a constant sperm concentration}

Table 4 shows that, at a constant sperm concentration, semen volume decreased as age increased (OR: 1.06; 95\% CI [1.04-1.08]; $p<0.0001)$. Sperm motility also decreased as age increased (OR: $1.07 ; 95 \%$ CI [1.04-1.09]; $p<0.0001)$. Therefore, each year the risk of presenting lower semen volumes increased 1.06 time over the previous year when concentration was kept constant. The risk of presenting impaired sperm motility increased 1.07 time each year when concentration was kept constant.

\section{tility}

Sperm volume and concentration at constant mo-

Table 5 shows that semen volume decreased as age increased when motility was kept constant (OR: $1.05 ; 95 \%$ CI [1.03-1.07]; $p<0.0001)$. Therefore, each year the risk 


\begin{tabular}{|c|c|c|}
\hline Semen parameters & OR (95\% CI) & $P$ value \\
\hline \multicolumn{3}{|l|}{ Volume } \\
\hline 31 to 40 years & $0.821(0.464-1.450)$ & 0.497 \\
\hline 41 to 50 years & $1.332(0.749-2.369)$ & 0.328 \\
\hline Over 50 years & $2.204(1.118-4.334)$ & 0.022 \\
\hline \multicolumn{3}{|l|}{ Sperm concentration } \\
\hline 31 to 40 years & $0.987(0.576-1.690)$ & 0.962 \\
\hline 41 to 50 years & $1.188(0.685-2.060)$ & 0.538 \\
\hline Over 50 years & $1.188(0.685-2.060)$ & 0.027 \\
\hline \multicolumn{3}{|l|}{ Total sperm count } \\
\hline 31 to 40 years & $2.462(0.985-6.150)$ & 0.054 \\
\hline 41 to 50 years & $2.926(1.160-7.381)$ & 0.023 \\
\hline Over 50 years & $6.151(2.261-16.738)$ & $<0.0001$ \\
\hline \multicolumn{3}{|c|}{ Sperm motility $(A+B)$} \\
\hline 31 to 40 years & $3.241(1.175-8.940)$ & 0.023 \\
\hline 41 to 50 years & $5.243(1.892-14.526)$ & 0.001 \\
\hline Over 50 years & $11.911(4.045-35.073)$ & $<0.0001$ \\
\hline \multicolumn{3}{|l|}{ Sperm morphology } \\
\hline 31 to 40 years & $1.810(0.433-7.556)$ & 0.416 \\
\hline 41 to 50 years & $2.395(0.567-10.119)$ & 0.235 \\
\hline Over 50 years & $3.223(0.654-15.866)$ & 0.150 \\
\hline \multicolumn{3}{|c|}{ DNA sperm fragmentation } \\
\hline 31 to 40 years & $1.243(0.364-4.240)$ & 0.728 \\
\hline 41 to 50 years & $1.388(0.399-4.829)$ & 0.606 \\
\hline Over 50 years & $4.583(1.167-17.999)$ & 0.029 \\
\hline
\end{tabular}

OR: Odds Ratio, CI: Confidence Interval

* Parameters were dichotomized in reference to the cutoffs defined by the WHO to categorize findings as normal or anomalous.

Table 3. Change in semen parameters at a constant semen volume

\begin{tabular}{|l|c|c|}
\hline Sperm motility & OR $(\mathbf{9 5 \%}$ CI) & p value \\
\hline Age & $1.060(1.038-1.083)$ & $<0.0001$ \\
\hline Sperm concentration & OR $(\mathbf{9 5 \%}$ CI $)$ & $\boldsymbol{p}$ value \\
\hline Age & $1.030(1.010-1.050)$ & 0.003 \\
\hline
\end{tabular}

OR: Odds Ratio,CI: Confidence Interval.

\begin{tabular}{|l|c|c|}
\hline \multicolumn{3}{|l|}{$\begin{array}{l}\text { Table 4. Change in semen parameters at a constant } \\
\text { sperm concentration }\end{array}$} \\
\hline Semen volume & OR (95\% CI) & $\boldsymbol{p}$ value \\
\hline Age & $\begin{array}{c}1.062 \\
(1.041-1.084)\end{array}$ & $<0.0001$ \\
\hline Sperm motility & OR (95\% CI) & $\boldsymbol{p}$ value \\
\hline Age & $\begin{array}{c}1.073 \\
(1.049-1.098)\end{array}$ & $<0.0001$ \\
\hline
\end{tabular}

OR: Odds Ratio,CI: Confidence Interval.

\begin{tabular}{|c|c|c|}
\hline Semen volume & OR (95\% CI ) & $p$ value \\
\hline Age & $\begin{array}{c}1.054 \\
(1.031-1.078)\end{array}$ & $<0.0001$ \\
\hline Sperm concentration & OR (95\% CI ) & $p$ value \\
\hline Age & $\begin{array}{c}0.992 \\
(0.957-1.027)\end{array}$ & 0.665 \\
\hline
\end{tabular}

OR: Odds Ratio,CI: Confidence Interval.

of presenting lower semen volumes increased 1.05 time over the previous year when motility was kept constant.

\section{DISCUSSION}

Aging is an inevitable process associated with multiple physiological changes, some of which affect the reproductive organs. This study focused on the effects of aging on semen parameters based on routine semen analyses and 
percent sperm DNA fragmentation. Although sperm parameters may change with aging, alterations do not necessarily correlate with male fertility outcomes. Nonetheless, other authors have attempted to find correlations between age and outcomes of assisted reproduction procedures (Das et al., 2013).

Tables 2, 3, 4 and 5 show the different semen parameters analyzed in our study. The tables show the relationships between age and semen parameters, describe the links between them, and report the risks assigned to them, which were combined to yield objective associations. Our findings suggested that semen volume, sperm concentration, total sperm count, and sperm motility correlated negatively with age. When compared to males aged 2130 years, men above the age of 50 were 2.2, 2.09, 6.15, and 11.91 times more likely to present lower semen volumes, lower sperm concentration, lower total sperm count, and impaired sperm progressive motility, respectively. Our findings were consistent with the data reported by Verón et al. (2018), thus confirming the effect of aging on semen parameters. They were also similar to the findings described by Stone et al. (2013), in which negative correlations between age and semen volume, sperm concentration, and progressive motility were derived. Although Oliveira et al. (2014) reported an association between age and semen volume and sperm progressive motility, the authors were unable to find a correlation between age and sperm concentration. However, Harris et al. (2011) emphasized the importance of sperm concentration and how spermatid concentration in seminiferous tubules decreased with age, thus accounting for the reduction of sperm concentration as age advances.

Sperm motility seems to be the parameter more significantly affected by age, since individuals in all age ranges presented a significantly increased risk of having anomalous findings compared to males aged $21-30$ years. Spermatozoa acquire motility in the prostate and epididymis. Therefore, the impairment observed with aging might be explained by the gradual decline individuals experience in endocrine function as they age. Harris et al. (2011) concluded that motility is affected with aging, in annual decreases of $0.17-0.8 \%$, which result in $3-16 \%$ decreases in motility over 20 years.

Differently from other authors (Neaves et al., 1984; Johnson et al., 1984; Harris et al., 2011), we were unable to find a statistically significant association between sperm morphology and age. Interestingly, Harris et al. (2011) reported that the most significant changes in semen quality are oligospermia, asthenospermia, and teratospermia, suggesting a progressive decline in normal sperm morphology equivalent to $0.2-0.9 \%$ per year of age. Other authors have reported that over a 20 -year period $4-18 \%$ of the spermatozoa present altered morphology (Auger et al., 1995; Andolz et al., 1999; Bujan et al., 1988). The disagreement between our findings and the studies of other authors might stem from changes in morphology assessment over the last few decades, disparities between study designs, or differences in statistical analysis.

Our study found a correlation between age and sperm DNA fragmentation. Males above the age of 50 presented a statistically significant increase in DNA damage and were 4.58 times more likely to present sperm DNA fragmentation than men aged 21-30. Other authors using the same technique described similar findings (Varshini et al., 2012; Oliveira et al., 2014). Petersen et al. (2018) also concluded that DNA fragmentation worsened with age and associated it with mitochondrial damage, since mitochondrial membrane potentials deteriorate significantly with age. One might argue that it is advisable to assess older men seeking fertility treatment for DNA fragmentation, since it may cause infertility (Ahmadi et al., 2016) and increase miscarriage rates (García-Ferreyra et al., 2015). Antioxidant therapy might be an option to treat men with sperm damage (Ahmadi et al., 2016).

When three variables were correlated (sperm concentration, volume, and motility), our data agreed with the previous analysis and showed increased semen parameter impairment with aging. A point to consider is that the data used in our study were collected from the male partners of infertile couples, a factor that might have introduced some selection bias. Our patients had been scheduled to undergo semen analysis and might represent a reproductively compromised population when compared to other men in general. In addition, our study did not control for other variables such as obesity, smoking, or alcohol drinking. These variables may also affect sperm parameters (García-Ferreyra et al., 2015; Oliveira et al., 2018). More studies with greater numbers of normal fertile individuals must be performed to confirm the effects of aging on sperm concentration, total sperm count, sperm motility, and sperm DNA fragmentation. Nevertheless, the findings reported in this and other similar studies (Varshini et al., 2012; Stone et al., 2013; Oliveira et al., 2014; Gunes et al., 2016; Petersen et al., 2018) suggest that aging has a negative effect on most semen parameters and DNA integrity. Therefore, age is an important factor to consider in the treatment of infertile couples.

\section{CONFLICTS OF INTEREST STATEMENT AND FUNDING/SUPPORT STATEMENT}

The authors have no conflict of interest to declare.

\section{Corresponding author:}

Víctor Pino

Faculty of Medicine

University of Los Andes.

Santiago, Chile.

E-mail: vmpino@miuandes.cl

\section{REFERENCES}

Ahmadi S, Bashiri R, Ghadiri-Anari A, Nadjarzadeh A. Antioxidant supplements and semen parameters: An evidence based review. Int J Reprod Biomed. 2016;14:729-36. PMID: 28066832

Andolz P, Bielsa MA, Vila J. Evolution of semen quality in North-eastern Spain: a study in 22,759 infertile men over a 36 year period. Hum Reprod. 1999;14:731-5. PMID: 10221705. DOI: $10.1093 /$ humrep/14.3.731

Auger J, Kunstmann JM, Czyglik F, Jouannet P. Decline in semen quality among fertile men in Paris during the past 20 years. N Engl J Med. 1995;332:281-5. PMID: 7816062. DOI: $10.1056 /$ NEJM199502023320501.

Bujan L, Mieusset R, Mondinat C, Mansat A, Pontonnier F. Sperm morphology in fertile men and its age related variation. Andrologia. 1988;20:121-8. PMID: 3389538 DOI: 10.1111/j.1439-0272.1988.tb00672.x

Crow JF. The origins, patterns and implications of human spontaneous mutation. Nat Rev Genet. 2000;1:40-7. PMID: 11262873 . DOI: $10.1038 / 35049558$

Das M, Al-Hathal N, San-Gabriel M, Phillips S, Kadoch IJ, Bissonnette $F$, Holzer $H$, Zini A. High prevalence of isolated sperm DNA damage in infertile men with advanced paternal age. J Assist Reprod Genet. 2013;30:843-8. PMID: 23722935. DOI: $10.1007 /$ s10815-013-0015-0 
Eisenberg DT. An evolutionary review of human telomere biology: the thrifty telomere hypothesis and notes on potential adaptive paternal effects. Am J Hum Biol. 2011;23:149-67. PMID: 21319244. DOI: 10.1002/ ajhb. 21127

Fernandez JL, Muriel L, Rivero MT, Goyanes V, Vazquez R, Alvarez JG. The sperm chromatin dispersion test: a simple method for the determination of sperm DNA fragmentation. J Androl. 2003;24:59-66. PMID: 12514084. DOI: 10.1002/j.1939-4640.2003.tb02641.x

García-Ferreyra J, Luna D, Villegas L, Romero R, Zavala $\mathrm{P}$, Hilario R, Dueñas-Chacón J. High Aneuploidy Rates Observed in Embryos Derived from Donated Oocytes are Related to Male Aging and High Percentages of Sperm DNA Fragmentation. Clin Med Insights Reprod Health. 2015;9:21-7. PMID: 26604851. DOI: 10.4137/CMRH. S32769

Goriely A, Wilkie AO. Paternal age effect mutations and selfish spermatogonial selection: causes and consequences for human disease. Am J Hum Genet. 2012;90:175200. PMID: 22325359. DOI: 10.1016/j.ajhg.2011.12.017

Gunes S, Hekim GN, Arslan MA, Asci R. Effects of aging on the male reproductive system. J Assist Reprod Genet. 2016;33:441-54. PMID: 26867640. DOI: 10.1007/ s10815-016-0663-y

Harris ID, Fronczak C, Roth L, Meacham RB. Fertility and the aging male. Rev Urol. 2011;13:e184-90. PMID: 22232567

Johnson L, Petty CS, Neaves WB. Influence of age on sperm production and testicular weights in men. J Reprod Fertil. 1984;70:211-8. PMID: 6694139 DOI: 10.1530/ jrf.0.0700211

Johnson SL, Dunleavy J, Gemmell NJ, Nakagawa S. Consistent age-dependent declines in human semen quality: a systematic review and meta-analysis. Ageing Res Rev. 2015;19:22-33. PMID: 25462195. DOI:10.1016/j. arr.2014.10.007

Kong $A$, Frigge $M L$, Masson $G$, Besenbacher $S$, Sulem $P$, Magnusson G, Gudjonsson SA, Sigurdsson A, Jonasdottir A, Jonasdottir A, Wong WS, Sigurdsson G, Walters GB, Steinberg S, Helgason H, Thorleifsson G, Gudbjartsson DF, Helgason A, Magnusson OT, Thorsteinsdottir U, Stefansson $\mathrm{K}$. Rate of de novo mutations and the importance of father's age to disease risk. Nature. 2012;488:471-5. PMID: 22914163. DOI: $10.1038 /$ nature 11396

Mahmoud AM, Goemaere S, El-Garem Y, Van Pottelbergh I, Comhaire FH, Kaufman JM. Testicular volume in relation to hormonal indices of gonadal function in community-dwelling elderly men. J Clin Endocrinol Metab. 2003;88:179-84. PMID: 12519849. DOI: 10.1210/jc.2002-020408
Neaves WB, Johnson L, Porter JC, Parker CR Jr, Petty CS. Leydig cell numbers, daily sperm production, and serum gonadotropin levels in aging men. J Clin Endocrinol Metab. 1984;59:756-63. PMID: 6434579. DOI: 10.1210/jcem$59-4-756$

Oliveira JBA, Petersen CG, Mauri AL, Vagnini LD, Baruffi RLR, Franco JG Jr. The effects of age on sperm quality: an evaluation of 1,500 semen samples. JBRA Assist Reprod. 2014;18:34-41. DOI: 10.5935/1518-0557.20140002

Oliveira JBA, Petersen CG, Mauri AL, Vagnini LD, Renzi A, Petersen B, Mattila M, Dieamant F, Baruffi RLR, Franco JG Jr. Association between body mass index and sperm quality and sperm DNA integrity. A large population study. Andrologia. 2018;50:e12889. PMID: 28856695. DOI: 10.1111/ and. 12889

Paniagua R, Amat P, Nistal M, Martin A. Ultrastructure of Leydig cells in human ageing testes. J Anat. 1986;146:17383. PMID: 1748904. DOI: 10.1002/jemt.1060190209

Petersen CG, Mauri AL, Vagnini LD, Renzi A, Petersen $B$, Mattila M, Comar V, Ricci J, Dieamant F, Oliveira JBA, Baruffi RLR, Franco JG. The effects of male age on sperm DNA damage: an evaluation of 2,178 semen samples. JBRA Assist Reprod. 2018;22:323-30. PMID: 30106542. DOI: 10.5935/1518-0557.20180047

Stone BA, Alex A, Werlin LB, Marrs RP. Age thresholds for changes in semen parameters in men. Fertil Steril. 2013;100:952-8. PMID: 23809502. DOI: 10.1016/j.fertnstert.2013.05.046

Varshini J, Srinag BS, Kalthur G, Krishnamurthy H, Kumar P, Rao SB, Adiga SK. Poor sperm quality and advancing age are associated with increased sperm DNA damage in infertile men. Andrologia. 2012;44:642-9. PMID: 22040161 DOI: $10.1111 / j .1439-0272.2011 .01243 . x$

Verón GL, Tissera AD, Bello R, Beltramone F, Estofan G, Molina RI, Vazquez-Levin MH. Impact of age, clinical conditions, and lifestyle on routine semen parameters and sperm kinematics. Fertil Steril. 2018;110:68-75.e4. PMID: 29980266. DOI: 10.1016/j.fertnstert.2018.03.016

World Health Organization (WHO). Laboratory Manual for the Examination and Processing of Human Semen. 5th ed. Geneva: World Health Organization; 2010.

Zitzmann M. Effects of age on male fertility. Best Pract Res Clin Endocrinol Metab. 2013;27:617-28. PMID: 24054934. DOI: $10.1016 /$ j.beem.2013.07.004 